\title{
Comprensión lectora en estudiantes universitarios
}

DOI: $10.46981 / \mathrm{sfjhv2n1-014}$

Received in: November 1st, 2020

Accepted in: December 30th, 2020

\author{
Jorge Guerra-García \\ Doctor en Psicología \\ Universidad Nacional Autónoma de México (Campus Iztacala) \\ Avenida de los Barrios \# 1, Col. Los Reyes Iztacala, Tlalnepantla, Estado de México, México. C.P. \\ 54090 \\ E-mail: guerra@unam.mx

\section{Carmen Yolanda Guevara-Benítez} \\ Doctora en Psicología \\ Universidad Nacional Autónoma de México (Campus Iztacala) \\ Avenida de los Barrios \# 1, Col. Los Reyes Iztacala, Tlalnepantla, Estado de México, México. C.P. \\ 54090 \\ E-mail: cyguevara@unam.mx
}

\begin{abstract}
RESUMEN
La comprensión lectora es una competencia genérica de relevancia para el estudiante, porque le permite obtener, aplicar y generalizar el conocimiento adquirido a diversas situaciones; sin embargo, los datos obtenidos de su evaluación muestran bajos desempeños en la población mexicana. Por consiguiente, resulta importante realizar estudios que aclaren el estado de la comprensión lectora en estudiantes universitarios mexicanos. El objetivo principal de este trabajo es describir la línea de investigación denominada Factores Asociados al Rendimiento Académico, que se ubica dentro del Grupo de Investigación en Aprendizaje Humano, de la Facultad de Estudios Superiores Iztacala-UNAM, y que se enfoca en aportar instrumentos, datos y evidencias acerca de la problemática mencionada. Aquí se exponen los objetivos de la línea, los principales resultados obtenidos hasta ahora y las investigaciones que se están realizando.
\end{abstract}

Palabras clave: Competencias genéricas, comprensión lectora, universitarios.

\begin{abstract}
Reading comprehension is a generic competence of relevance for students, because it allows them to obtain, apply and generalize the knowledge acquired to different situations; however, the data obtained from its evaluation show low performance in the Mexican population. Therefore, it is important to conduct studies that clarify the state of reading comprehension in Mexican university students. The main objective of this paper is to describe the research line called Factors Associated with Academic Performance, which is located within the Research Group on Human Learning, of the Facultad de Estudios Superiores Iztacala-UNAM, and which focuses on providing instruments, data and evidence about the aforementioned problem. The objectives of the line, the main results obtained so far and the research being carried out are presented here.
\end{abstract}

Key words: Generic competencies, reading comprehension, university students. 


\section{COMPETENCIAS}

Una competencia implica que una persona integre una serie de elementos (conocimientos, técnicas, actitudes, procedimientos y valores) para demostrar que puede resolver una situación problemática concreta (Villa \& Poblete, 2011). Las competencias pueden estar circunscritas a condiciones particulares que respondan a un dominio concreto de estudio o de trabajo, a estos casos se les ha denominado competencias específicas; o bien, pueden ser transferibles, es decir, responder eficientemente a circunstancias y contextos desligados de aquellos en los que las competencias fueron originalmente aprendidas y utilizadas, en estos casos se nombran competencias genéricas (Beneitone, Esquetini, González, Marty, Siufi \& Wagenaar, 2007).

\section{LA COMPRENSIÓN LECTORA COMO COMPETENCIA GENÉRICA}

El aprendizaje de las competencias genéricas es indispensable en el contexto de la educación superior, dado que aumenta en el estudiante la probabilidad de un mayor desarrollo académico y un relativamente fácil ingreso al campo laboral. En este sentido, la comprensión lectora constituye un excelente ejemplo de competencia genérica, dado que se aplica para adquirir conocimientos de diversa índole, y aquellos conocimientos adquiridos se pueden transferir a situaciones problemáticas extraacadémicas y novedosas (Guevara \& Guerra, 2013).

En las últimas dos décadas, particularmente en países de América Latina, el estudio de la comprensión lectora ha adquirido relevancia, entre otras razones, debido a los resultados insatisfactorios que se han obtenido en evaluaciones internacionales de estudiantes latinoamericanos, por ejemplo, las aplicadas por la Organización para la Cooperación y el Desarrollo Económico (OECD, por sus siglas en inglés) denominadas PISA. Los resultados de la aplicación de esta prueba en 2015, muestran que la población objetivo (estudiantes de 15 años de edad), de los países de América Latina que participaron, obtuvieron un puntaje por debajo del promedio, en la prueba de comprensión lectora. El rendimiento promedio de los alumnos, considerando los 35 países integrantes de la OECD fue de 493 puntos, con una desviación estándar de 96 puntos. México obtuvo 423 puntos, solo por arriba de Brasil (407) y Perú (398), mientras que se colocó por debajo de Colombia (425), Trinidad y Tobago (427), Costa Rica (427), Uruguay (437), Chile (459) y Argentina (475) (OECD, 2016). La más reciente aplicación de la citada prueba arrojó resultados similares. Todos los países participantes de América Latina tuvieron un rendimiento menor al promedio de la OCDE (2019), el cual fue de 487 puntos. México obtuvo 420 puntos, solo quedaron por arriba de él, Chile (452), Uruguay (427) y Costa Rica (426); mientras que, por debajo, se ubicaron Brasil (413), Colombia (412), Argentina (402), Perú (401) y Panamá (377). 


\section{IMPORTANCIA DE LA COMPRENSIÓN LECTORA}

La adquisición y uso generalizado de la comprensión lectora es de suma importancia en los niveles educativos superiores, dado el hecho de que una gran parte de la formación de los alumnos universitarios se lleva a cabo a través de la lectura de textos.

La revisión de la literatura latinoamericana respecto a la problemática muestra que los estudiantes universitarios se desempeñan deficientemente en diversos niveles de comprensión. Se identifican como principales dificultades: la selección y jerarquización de ideas principales, el uso de la inferencia para deducir información que no se encuentra de forma explícita en el texto, la utilización del conocimiento previo para relacionarlo con lo leído, así como la realización de un juicio valorativo respecto a los contenidos revisados, en donde se contrasten las afirmaciones del escrito con las propias (Cisneros, Olave \& Rojas, 2010; Echevarría \& Gastón, 2000; Guerra, 2014). Por lo anterior, resulta importante realizar estudios que aporten datos del estado de la comprensión lectora en estudiantes universitarios mexicanos. El objetivo principal de este trabajo es describir la línea de investigación denominada "Factores asociados al rendimiento académico" que se ubica dentro del Grupo de Investigación en Aprendizaje Humano, de la Facultad de Estudios Superiores Iztacala-UNAM.

\section{OBJETIVOS DE LOS ESTUDIOS DE NUESTRA LÍNEA DE LNVESTIGACIÓN}

La línea de investigación denominada Factores asociados al rendimiento académico se ubica dentro del Grupo de Investigación en Aprendizaje Humano de la Facultad de Estudios Superiores Iztacala-UNAM. Desde 2014, ha realizado diversos trabajos cuyos objetivos se han centrado en: a) desarrollar diversos instrumentos que evaluen la comprensión lectora, el uso de estrategias lectoras, la motivación hacia la lectura y otras variables asociadas; b) describir y relacionar el nivel de comprensión lectora y el uso de estrategias con diversas variables sociodemográficas y académicas en estudiantes universitarios; c) identificar la relación que guardan el tiempo invertido en la lectura, la comprensión y el uso de estrategias, y c) fomentar el uso de estrategias lectoras en la población universitaria.

\section{PRINCIPALES RESULTADOS}

\subsection{DISEÑO DE UN INSTRUMENTO PARA MEDIR COMPRENSIÓN LECTORA EN ALUMNOS} UNIVERSITARIOS (ICLAU).

Para la elaboración del instrumento se partió de la conceptualización de la comprensión lectora como el proceso mediante el cual el lector, a través del uso de diversas estrategias, interactúa con el texto en distintos niveles de competencia (Pérez, 2005). El instrumento consta de un texto de tipo expositivoargumentativo denominado La evolución y su historia, extraído de la obra de Cela y Ayala (2001), que es una narración de 965 palabras cuyo contenido explica la evolución biológica, haciendo énfasis en sus 


\section{Tabla 1. Niveles de comprensión, medición y criterios de evaluación del ICLAU}

Nivel Se identifica cuando el lector:

Literal Reconoce y recuerda, directamente del texto, las ideas tal y como las expresa el
autor.

Reorganización de la Ordena las ideas mediante procesos de clasificación y síntesis; por ejemplo, información cuando reseña, resume o sintetiza la lectura de un texto con sus propias palabras o cuando lo expresa gráficamente a través del uso de conceptos vinculados por símbolos que indican relaciones, jerarquías, etcétera.

$\begin{array}{ll}\text { Inferencia } & \text { Agrega elementos que no están en el texto, para relacionarlo con sus } \\ \text { experiencias personales o para deducir ideas que no están explícitas en el } \\ \text { escrito, posibilitando de esta manera su interpretación. }\end{array}$

Crítico

Utiliza procesos de valoración. Necesita establecer una relación entre lo que dice el texto y el conocimiento previo que tiene sobre el tema, para que luego evalúe las afirmaciones del escrito contrastándolas con las propias.

Apreciación

Expresa comentarios emotivos o estéticos sobre el texto consultado, o puede emitir juicios sobre su particular estilo literario o sobre el uso o características del lenguaje que utiliza el autor; por ejemplo, el empleo de la ironía, del humor, del doble sentido, etcétera.

El procedimiento típico para evaluar comprensión lectora por medio del ICLAU consiste en proporcionarle al alumno, de forma individual o grupal, el texto a leer junto con siete reactivos, sin restricciones de tiempo. La calificación de la prueba se establece principalmente a través de rúbricas. El proceso de diseño y validación con estudiantes universitarios mexicanos fue reportado por Guerra y Guevara (2013).

\subsection{DISEÑO DE UN INVENTARIO DE ESTRATEGIAS METACOGNOSCITIVAS Y MOTIVACIÓN POR LA LECTURA (IEMML).}

El diseño del inventario partió de la definición de estrategias como el conjunto de acciones que regulan el comportamiento lector de los estudiantes, permitiéndoles seleccionar, evaluar, persistir o abandonar dichas acciones, en función de lograr o no la comprensión (Peña, 2000). El inventario consta de 27 reactivos que se responden con base en una escala tipo Likert de cinco categorías (nunca, rara vez, 
a veces, frecuentemente y siempre). Se evalúan: a) estrategias de análisis de la lectura, que incluye relacionar los contenidos de un texto con otros previamente revisados o con experiencias vividas, parafrasear lo leído, reflexionar sobre el tema, elaborar nuevas ideas y conclusiones; b) estrategias para identificación de información o de ideas, que contiene reactivos referentes a conocer el significado de los términos utilizados en el texto, ubicar las ideas principales; c) estrategias de consulta de fuentes de información, para evalúan la búsqueda del significado de palabras y la consulta de nuevas fuentes bibliográficas; d) motivación intrínseca por la lectura en general, donde se plantean aseveraciones relacionadas con actividades de lectura, realizadas por iniciativa propia, de cualquier tipo de texto, y e) motivación intrínseca por la lectura de textos académicos, cuyos reactivos se relacionan con el interés de los alumnos hacia este tipo de textos. El inventario fue diseñado, validado y reportado por Guerra, Guevara y Robles (2014).

\subsection{EVALUACIÓN DE LOS NIVELES DE LA COMPRENSIÓN LECTORA EN ESTUDIANTES UNIVERSITARIOS.}

Se llevó a cabo un estudio en el que participaron 570 estudiantes de la Carrera de Psicología, de primero, tercero, quinto y séptimo semestres; su edad promedio fue de 19.9 años. Se consideró como unidad de análisis el grupo escolar en el que estaban inscritos los participantes, del turno matutino y vespertino. Se utilizó un muestreo probabilístico estratificado de tipo proporcional, considerando semestre y turno. Se procedió de la misma forma que la señalada anteriormente en el diseño del ICLAU. Los resultados de la muestra total revelaron un porcentaje promedio general de $66 \%$ de respuestas correctas. Los tres niveles de comprensión que mostraron porcentajes inferiores a 50\% fueron: literal, inferencial y crítico; mientras que los niveles de organización de la información y apreciativo exhibieron porcentajes por encima del 70\%. En cuanto a la ejecución de los alumnos en función del semestre estudiado, un análisis ANOVA de un factor, arrojó diferencias significativas, al nivel de $p<.05$, en cuatro niveles de comprensión: literal, inferencial, crítico y apreciativo, en lo general, a favor de los alumnos de los primeros semestres. Los resultados referentes al bajo nivel de comprensión son congruentes con otros estudios que se han realizado en el área geográfica de Latinoamérica. Este trabajo de investigación ha sido reportado por Guevara, Guerra, Delgado y Flores (2014).

\subsubsection{Comparación de la comprensión lectora en distintas carreras universitarias.}

Para agregar datos a los reportados previamente, obtenidos con alumnos de la Carrera de Psicología, se llevó a cabo otro estudio para evaluar los niveles de comprensión lectora en estudiantes de Matemáticas Aplicadas y Computación, de una universidad pública de la Ciudad de México, utilizando el ICLAU. Participaron 309 estudiantes, de los cuales $69 \%$ fueron de primer ingreso, mientras 
que $31 \%$ pertenecían al último semestre de la carrera. Los resultados encontrados revelaron que los estudiantes de la muestra contaban con bajos niveles de comprensión, en vista de que solo pudieron contestar de forma correcta un 53\% de la prueba. El nivel de comprensión que obtuvo el porcentaje más alto fue el inferencial, con $87 \%$ de respuestas acertadas. Estos datos pueden revelar el posible impacto de los planes y programas académicos, sobre niveles específicos de la comprensión lectora; suposición que se encuentra en vías de aportar evidencias. Esta investigación se encuentra en proceso de publicación.

Un estudio más estuvo encaminado a evaluar los niveles de comprensión lectora en estudiantes de la carrera de Biología. Se aplicó el ICLAU a una muestra intencional de 54 alumnos de segundo semestre, de una universidad pública. Sus edades fluctuaron entre los 18 y 25 años; $59 \%$ eran mujeres y $41 \%$ hombres. Los resultados mostraron un bajo nivel de comprensión lectora, dado que respondieron de forma correcta sólo el $45 \%$ de la prueba. Los dos niveles de comprensión más altos fueron el literal con $63 \%$ de aciertos y el inferencial con $62 \%$, mientras que el más bajo fue el nivel crítico con $32 \%$. Estos resultados deben ser tomados con cautela debido al tamaño de la muestra y a su forma de selección con carácter intencional.

Sin embargo, los hallazgos pueden explicarse -al igual que en el estudio anterior- haciendo referencia a los contenidos curriculares de los programas académicos y, también, pueden guardar relación con el tipo de actividades demandadas por los profesores, todo ello en el marco de la carrera universitaria que los alumnos cursan. Por lo tanto, resulta necesario investigar a profundidad aspectos tales como: el tipo de objetivos (de conocimiento, de análisis, de aplicación, etcétera) de los programas académicos, la clase de demandas o tareas que realizan los alumnos por exigencia de los profesores, entre otros aspectos, para tener evidencia que fundamente la anterior hipótesis. Los resultados de este estudio también se encuentran en proceso de publicación.

\subsection{USO DE ESTRATEGIAS LECTORAS Y MOTIVACIÓN HACIA LA LECTURA EN ESTUDIANTES DE DISTINTAS CARRERAS UNIVERSITARIAS.}

Teniendo como objetivo conocer y comparar las estrategias metacognoscitivas y el nivel de motivación hacia la lectura, se aplicó el IEMML, a través de una plataforma digital, a 900 estudiantes de primer semestre de seis carreras (Biología, Enfermería, Medicina, Odontología, Optometría y Psicología) de una universidad pública mexicana. Los puntajes obtenidos en el total del IEMML, así como en cada uno de sus factores, se ubicaron en cinco categorías, con base en rangos equidistantes: muy alto, alto, medio, bajo y muy bajo. El puntaje total del IEMML obtuvo un promedio de 96, lo cual indica que los estudiantes reportaron un uso frecuente de las estrategias evaluadas y una motivación alta hacia la lectura. La carrera con mayor promedio (100 puntos) fue Biología y la de menor promedio (90 
puntos) fue Optometría. En cuanto al tipo de estrategias, las ubicadas en análisis de la lectura todos los estudiantes se encuentran en un nivel alto; sin embargo, las de identificación de la información y de consulta de fuentes adicionales, así como en los dos tipos de motivación hacia la lectura de textos (generales y académicos), varias carreras muestran niveles medios. Las carreras de Odontología y Optometría son las que obtuvieron menores niveles en el uso de estrategias y en motivación hacia la lectura. Estos resultados fueron reportados por Guerra, Guevara, Rugerio y Hermosillo (2018) y Guerra, Guevara y Rugerio (2019).

\subsection{APLICACIÓN DE UN CURSO-TALLER PARA FOMENTAR LA COMPRENSIÓN LECTORA} EN UNIVERSITARIOS.

Se diseñó y aplicó un Curso-taller a estudiantes universitarios, con el objetivo de medir sus efectos sobre la comprensión lectora, el uso de estrategias y la motivación por la lectura. Participaron 13 alumnos de cuarto semestre de la carrera de Psicología, de una universidad pública mexicana. La edad promedio de los estudiantes fue de 21 años, y $92 \%$ de ellos fueron de sexo femenino. Se utilizó un diseño pretest-postest en donde antes y después de la intervención se aplicaron el ICLAU y el IEMML para comparar los puntajes de las variables comprensión, estrategias y motivación hacia la lectura. El Curso-taller consistió en promover el uso de estrategias lectoras vinculadas a niveles específicos de comprensión: literal, reorganización de información, inferencial, crítico y apreciativo. El procedimiento típico fue el siguiente: primero, se proyectaba un vídeo explicativo relacionado con el nivel de comprensión lectora a practicar; después, los facilitadores llevaban a cabo una exposición introductora que incluía las características que lo componen, los procesos psicológicos implicados y las diversas estrategias sugeridas para alcanzarlo. Posteriormente, se instruía de forma directa sobre cada una de ellas; enseguida, se les proporcionaban a los alumnos pequeños textos y ejercicios en donde pudieran aplicarlas. Finalmente, se les brindaba retroalimentación sobre la actividad en su totalidad. Los resultados del Postest mostraron que 12 de los 13 participantes tuvieron un incremento en algún nivel de la comprensión lectora, aunque no fue suficiente para obtener diferencias estadísticamente significativas entre los puntajes de pretest y postest. En relación con las estrategias de comprensión y motivación hacia la lectura, al comparar los puntajes obtenidos en el IEMML antes y después de la aplicación del Curso-taller, aquellas relacionadas con el análisis de la información y con la consulta de fuentes adicionales, obtuvieron diferencias significativas $(\mathrm{Z}=-2.521, p=.012$ y $\mathrm{Z}=-2.754, p=.006$, respectivamente); de igual forma, la motivación por la lectura en general $(\mathrm{Z}=-2.944, p=.003)$.

Este trabajo aporta evidencias que contribuyen al conocimiento sobre el uso de estrategias efectivas para la comprensión lectora, y se suma a los escasos estudios vinculados con el fomento explícito de la comprensión lectora en estudiantes universitarios, a través de la enseñanza directa de 
estrategias idóneas a los diferentes niveles de comprensión.

\section{INVESTIGACIONES EN CURSO}

Actualmente se está investigando si las características de los programas académicos que cursan los estudiantes fomentan algún tipo específico de nivel de comprensión lectora. Para esto, se pretende evaluar la comprensión lectora y sus diferentes niveles y, luego, analizar los programas académicos en función de: (a) el tipo de objetivos (de conocimientos, de análisis, de aplicación, etcétera), tanto generales como específicos, que se pretenden conseguir, (b) el tipo de conocimientos y habilidades que el profesor demanda de sus alumnos, (c) las actividades o tareas que frecuentemente realizan los alumnos para acreditar la asignatura, entre otras.

Por último, es importante señalar que, para nuestro grupo de investigación, el instrumento ICLAU ha sido efectivo para evaluar la comprensión lectora, sin embargo, no puede ser utilizado como pretest y postest en investigaciones dirigidas a la enseñanza de estrategias lectoras en los alumnos universitarios que lo requieran. Por tal motivo, se hace necesario contar con una segunda versión del instrumento (ICLAU-2), que sea equivalente a la existente; una de las versiones se podrá utilizar antes del programa de intervención y la otra después del programa.

Por lo anterior, uno de nuestros actuales objetivos de investigación es elaborar y validar un instrumento paralelo al ICLAU, para evaluar la comprensión lectora de estudiantes universitarios de diferentes disciplinas. Dado que la primera versión del instrumento aborda un tema de Biología (Evolución), se elaboró un texto sobre Genética, que es un tema de la misma disciplina científica, para lo cual se requirió consultar diversas fuentes bibliográficas. También se han conformado los reactivos para el ICLAU-2. Actualmente, el instrumento se encuentra en un proceso de valoración por parte de jueces expertos. 


\section{REFERENCIAS}

Beneitone, P., Esquetini, C., González, J., Maletá, M., Siufi, G. \& Wagenaar, R. (2007). Reflexiones y perspectivas de la Educación Superior en la América Latina. España: Universidad de Deusto.

Cela, C. C. J. \& Ayala, F. J. (2001). Senderos de la evolución humana. Madrid: Alianza.

Cisneros, E. M., Olave, A. G. \& Rojas, G. I. (2010). La inferencia en la comprensión lectora. De la teoría a la práctica en la Educación Superior. Pereira: Universidad Tecnológica de Pereira.

Echevarría, M. A. \& Gastón, B. I. (2010). Dificultades de comprensión lectora en estudiantes universitarios. Implicaciones en el diseño de programas de intervención. Revista de Psicodidáctica 11(2), 169-188.

Guerra, G. J. (2014). Competencias genéricas en estudiantes de educación superior: el caso de la comprensión lectora. Tesis para obtener el grado de doctor en Psicología. México: Universidad Nacional Autónoma de México.

Guerra, J., Guevara, Y., López, A. \& Rugerio, J. P. (2014). Identificación de las estrategias y motivación hacia la lectura, en estudiantes universitarios mexicanos. CPU-e Revista de Investigación Educativa, 19, 254-277. ISSN 1870-5308.

Guerra, J., Guevara, Y. \& Robles, S. (2014). Validación del Inventario de Estrategias Metacognoscitivas y Motivación por la Lectura (IEMML) en estudiantes de psicología. USB Psicología-Revista Psicogente 17(31), 17-32. ISSN 0124-0137

Guerra, J., Guevara, Y. y Rugerio, J. P. (2019). Aportaciones a la descripción del estudiantado de la Facultad de Estudios Superiores Iztacala. Revista Electrónica de Psicología Iztacala, 22(3), 2574-2600. ISSN 1870-8420.

Guerra, J., Guevara, Y., Rugerio, J. P. \& Hermosillo, A. (2018). Estrategias lectoras y motivación hacia la lectura en estudiantes de distintas carreras universitarias. Revista Mexicana de Investigación en Psicología 10(1), 17-29.

Guevara, B. Y. \& Guerra, G. J. (2013). La comprensión lectora como competencia genérica. Revista Electrónica de Psicología Iztacala 16(2), 319-339.

Guevara, Y., Guerra, J., Delgado, U. y Flores, C. (2014). Evaluación de distintos niveles de comprensión lectora en estudiantes mexicanos de psicología. Revista Acta Colombiana de Psicología 17(2), 113-121. ISSN 0123-9155

OECD (2016). Pisa 2015 Results (Volume I): Excellence and Equity in Education. Paris: OECD. http://dx.doi.org/10.1787/9789264266490-en

OECD (2019). Nota país. Programa para la Evaluación Internacional de Alumnos (PISA), PISA 2018Resultados. Volúmenes I-III, 1-12. http://www.oecd.org/pisa/

Peña, G. J. (2000). Las estrategias de lectura: su utilización en el aula. Educere 4(11), 159-163.

Pérez, Z. M. J. (2005). Evaluación de la comprensión lectora: dificultades y limitaciones. Revista de Educación. Número Extraordinario. 121-138. 
Villa, S. A. \& Poblete, R. M. (2011). Evaluación de competencias genéricas: principios, oportunidades y limitaciones. Bordón. Revista de Pedagogía 63(1), 147-170. 